\title{
OPTIMAL LOCAL SMOOTHING AND ANALYTICITY RATE ESTIMATES FOR THE GENERALIZED NAVIER-STOKES EQUATIONS *
}

\author{
HONGJIE DONG ${ }^{\dagger}$ AND DONG LI $^{\ddagger}$
}

\begin{abstract}
By using a new bilinear estimate, a pointwise estimate of the generalized Oseen kernel, and an idea of a fractional bootstrap, we establish optimal local smoothing and decay estimates of solutions to the Navier-Stokes equations with fractional dissipation. We also show that solutions are analytic in space variables.
\end{abstract}

Key words. Spatial analyticity, bootstrap, Navier-Stokes equations.

AMS subject classifications. 35Q30,35Q35,35B65.

\section{Introduction}

We are interested in the initial value problem of $d$-dimensional generalized NavierStokes equations with fractional dissipation

$$
\left\{\begin{array}{l}
u_{t}+u \nabla u+(-\Delta)^{\gamma / 2} u+\nabla p=f, \quad \operatorname{div} u=0 \\
u(0, x)=u_{0}(x) \quad x \in \mathbb{R}^{d}
\end{array}\right.
$$

where $d \geq 2, f$ is the external force, $\gamma>0$ is a fixed parameter, and the initial data $u_{0}$ is in some Banach space to be specified later. Here $(-\Delta)^{\gamma / 2} u$ is defined by its Fourier transform $\left(\widehat{-\Delta)^{\gamma / 2}} u=|\xi|^{\gamma} \hat{u}\right.$. The generalized equations (1.1) have been studied by many authors. See, for instance, [21, 12, 27] and [28].

When $\gamma=2$, (1.1) reduces to the classical incompressible Navier-Stokes equations. In a well-known paper [13], Kato proved that for $\gamma=2$ the problem is locally wellposed for $u_{0} \in L^{d}$. Kato's method is based on the perturbation theory of the Stokes kernel, and the so called mild solutions are constructed via a fixed point argument by considering the corresponding integral equations. His results have been generalized by many authors in various function spaces (see, for example, Koch-Tataru [15], and references therein). With minor modifications, this method can also be applied to show local well-posedness of the generalized Navier-Stokes equations (1.1) with initial data $u_{0} \in L^{\frac{d}{\gamma-1}}$ and global well-posedness for small data (see Proposition 2.1).

In [22], Masuda initiated the study of the spatial analyticity of solutions to the Navier-Stokes equations. The temporal analyticity was proved by Foias and Temam in an important paper [5]. By using a certain time-dependent weight, they showed that solutions to the Navier-Stokes equations on the torus are analytic in time with values in a Gevrey class of functions. With a smallness assumption, their method can be adapted to deal with the equations in the whole space (see, for example, LemariéRieusset $[18,19]$ and $[20])$. The study of analyticity of the Navier-Stokes equations was continued by many authors. In a very interesting paper [11], Kahane established the spatial analyticity of weak solutions in Serrin's class $L_{t}^{p} L_{x}^{q}$ with $d / q+2 / p<1$.

${ }^{*}$ Received: July 31, 2008; accepted (in revised version): November 26, 2008. Communicated by Francois Golse.

${ }^{\dagger}$ Division of Applied Mathematics, Brown University, 182 George Street, Box F, Providence, RI 02912, USA (Hongjie_Dong@brown.edu).

${ }^{\ddagger}$ School of Mathematics, Institute for Advanced Study, Einstein Drive, Princeton, NJ 08540, USA (dongli@math.ias.edu). 
For equations in cylindrical domains, Komatsu [16] extended the work by Masuda and Kahane by showing that the solutions have global spatial analyticity up to the boundary. By using tools in complex analysis, Grujić and Kukavica [9] proved that with initial data in $L^{p}, p>d$, there exists a local analytic solution of the Navier-Stokes equations in $C\left([0, T), L^{p}\left(\mathbb{R}^{d}\right)\right)$ for some $T>0$. Optimal local smoothing and decay estimates of mild solutions with initial data in scale-invariant spaces $L^{d}$ or $\dot{H}^{d / 2-1}$ were established recently by Giga and Sawada [8] and Sawada [26]. In particular, in a very recent paper [24], Miura and Sawada showed that the solutions by Koch and Tataru [15] are spatial analytic. A similar optimal smoothness result is also obtained independently in a recent paper [7] by Germain, Pavlović, and Staffilani for both the $L^{\infty}$ and the Carleson norms.

For the generalized Navier-Stokes equations, in [21] Lions proved that in three space dimensional case for any $\gamma \geq 5 / 2$, equations (1.1) always possess global classical solutions. With $\gamma>1$, the local existence and uniqueness results of (1.1) in Besov spaces were established recently in Wu [27]. In an interesting paper [12] Katz and Pavlović showed a partial regularity result of Caffarelli-Kohn-Nirenberg's type: in the three space dimensional case the Hausdorff dimension of the singular set of (1.1) at the time of first blow-up is at most $5-2 \gamma$.

We remark that usually local smoothing estimates of solutions to the classical Navier-Stokes equations are obtained by using either the fixed point argument (the contraction argument, see, for example, $[8,26,24]$ and [7]) or a variation of Foias and Temam's method (see, for example, [19]). For both methods, one always needs some kind of a smallness assumption on either the initial data or the solution itself.

To the best of our knowledge, the local smoothing and analyticity rate estimates for the generalized Navier-Stokes equations have not been studied in detail. It seems to us that the proofs in $[8,26,24]$ or [7] cannot be easily extended to the generalized Navier-Stokes equations when $\gamma<2$ (see Remark 2.4). On the other hand, when $\gamma<1$, even solutions to the corresponding linear equations are not spatially analytic ${ }^{1}$. Thus one cannot expect this for nonlinear equations.

In this paper, we focus on the case $\gamma \in(1,2]$, which generalizes the more physical case $\gamma=2$. The main results (Theorem 2.1 and 2.5) show that spatial analyticity is an intrinsic property of the solutions to the generalized Navier-Stokes equations. We show that the existence of the solution in certain path spaces implies its smoothness and analyticity without any smallness assumption. Our estimates are optimal and we give a simplified proof of a result of the classical Navier-Stokes equations by Giga and Sawada [8]. The approach used here is more direct than some previous work by using the aforementioned contraction argument (see Remark 2.4). The method is based on new estimates of the kernels and a so-called fractional bootstrap argument.

Our treatment of the bilinear term makes better use of the smoothing effect of the Oseen kernel. The idea, roughly speaking, is that the smoothing effect should take place gradually in time (see section 4 ). We use the fractional bootstrapping by noticing that in the subcritical case one can put a little bit more than one derivative on the kernel and still keep the convergence of the integral of the bilinear term. The advantage is that we can avoid using the contraction argument as long as the solution is known to exist in certain path spaces. Furthermore, the estimate of fractional derivatives can also be obtained almost immediately. This also explains why it is called fractional bootstrapping, since at each step the increment of regularity is a fraction rather than an integer. Another idea of the proof is to use the semigroup property of

\footnotetext{
${ }^{1}$ This fact can be verified by directly computing the derivatives of the kernel at the origin.
} 
the mild solutions, which enables us to simplify the corresponding argument in [3].

The remaining part of the article is organized as follows: the main results are given in the following section. Section 3 is devoted to a new pointwise estimate of the generalized Oseen kernel and a corresponding estimate of the generalized heat kernel. In section 4 we prove Theorem 2.1 by mainly using the aforementioned fractional bootstrap argument. Finally, the proof of Theorem 2.5 is given in the last section.

\section{The main results}

Before stating the main results, we introduce some notation. Define $G_{\gamma}(t, x)$ by its Fourier transform $\widehat{G_{\gamma}}(t, \xi)=e^{-t|\xi|^{\gamma}}$ for $t>0$. Then $G_{\gamma}(t, x)$ is the fundamental solution of the linear operator $\partial_{t}+(-\Delta)^{\gamma / 2}$ and it has the scaling property

$$
G_{\gamma}(t, x)=t^{-\frac{d}{\gamma}} G_{\gamma}\left(1, t^{-\frac{1}{\gamma}} x\right) .
$$

It is well-known that (1.1) has an integral formulation:

$$
u(t)=G_{\gamma}(t, \cdot) * u_{0}-\int_{0}^{t} K_{\gamma}(t-r, \cdot) *(u \nabla u-f)(r, \cdot) d r,
$$

where $\mathcal{P}$ is the Helmholtz projection, and $K_{\gamma}=\mathcal{P} G_{\gamma}$ is the Oseen kernel (see section 3 and Proposition 3.1).

For any $p \in(1, \infty)$, denote $E^{p}$ to be the closure of

$$
\left\{u \in C_{0}^{\infty}\left(\mathbb{R}^{d}\right) ; \operatorname{div} u=0\right\}
$$

in $L^{p}\left(\mathbb{R}^{d}\right)$. For $q \in\left(\frac{d}{\gamma-1}, \infty\right], T \in(0, \infty]$, introduce the Banach spaces

$$
X_{q, T}=C\left([0, T), E^{\frac{d}{\gamma-1}}\right) \cap\left\{u \mid t^{\alpha} u \in C\left((0, T), L_{x}^{q}\right)\right\}, \quad \alpha=1-\frac{1}{\gamma}-\frac{d}{q \gamma},
$$

with norm

$$
\|u\|_{X_{q, T}}=\max \left\{\|u\|_{L_{t}^{\infty}([0, T)) L_{x}^{\frac{d}{\gamma-1}}},\left\|t^{\alpha} u\right\|_{L_{t}^{\infty}((0, T)) L_{x}^{q}}\right\} .
$$

We assume that $f$ satisfies

$$
\left\|D_{x}^{k} \mathcal{P} f(t, \cdot)\right\|_{L_{x}^{\frac{d}{\gamma-1}}} \leq C^{k+1} t^{-\frac{k}{\gamma}}
$$

for some $C>0$, any $k \geq 0$, and $t \in(0, \infty)$. In particular, all conservative ${ }^{2}$ functions are included.

The classical Kato's method [13] easily gives the following local well-posedness result.

Proposition 2.1. Let $\gamma \in(1,2]$. Suppose the initial data $u_{0}$ is in the scaling invariant space $E^{\frac{d}{\gamma-1}}\left(\mathbb{R}^{d}\right)$. Then for any $q \in\left(\frac{d}{\gamma-1}, \infty\right]$, equation $(2.2)$ has a unique solution $u$ in $X_{q, T}$ for some $T \in(0, \infty]$. Here $T$ can be chosen to depend continuously on $u_{0}$ in $E^{\frac{d}{\gamma-1}}-$ topology.

Here we state our main results of this note.

\footnotetext{
${ }^{2}$ Here we say $f$ is conservative if $\mathcal{P} f \equiv 0$, where $\mathcal{P}$ is the Helmholtz projection.
} 
Theorem 2.1. Let $\gamma \in(1,2]$. Suppose $u$ is a solution to $(2.2)$ in $\mathbb{R}^{d} \times(0, T)$ for some $T \in(0, \infty)$ and satisfies

$$
\left\|t^{\alpha} u\right\|_{L_{t}^{\infty}((0, T)) L_{x}^{q}}<\infty
$$

for some $q \in\left(\frac{d}{\gamma-1}, \infty\right)$ and $\alpha=1-\frac{1}{\gamma}-\frac{d}{q \gamma}$. Then for any $t \in(0, T), q^{\prime} \in[q, \infty]$, and $k=0,1,2, \cdots$, we have

$$
\left\|D_{x}^{k} u(t, \cdot)\right\|_{L_{x}^{q^{\prime}}} \leq C^{k+1} t^{-\frac{k}{\gamma}-\alpha^{\prime}} k^{k},
$$

where $\alpha^{\prime}=1-\frac{1}{\gamma}-\frac{d}{q^{\prime} \gamma}$ and $C$ is independent of $k$ and $q^{\prime}$. Consequently, $u(t, \cdot)$ is spatially analytic.

REMark 2.2. If $f$ is conservative, i.e., $\mathcal{P} f \equiv 0$, the time $T$ in Theorem 2.1 may be infinite. In that case, estimate (2.4) implies the decay in time of higher order Sobolev norms. Furthermore, the radius of convergence of Taylor's expansion of $u(t, \cdot)$ increases with time at a rate proportional to $t^{1 / \gamma}$.

REMARK 2.3. As was already mentioned, one feature of Theorem 2.1 is that no assumption is made on the size of the initial data $u_{0}$. In other words, the mere existence of the solution implies its analyticity. This is accomplished by using fractional bootstrapping (see section 4) instead of the classical contraction argument. We emphasize that our result is conditional and depends on the existence of solutions in certain path spaces.

For other results of similar type, we refer the reader to Gallagher, Iftimie, and Planchon [6], where the authors established a decay of solutions to the Navier-Stokes equations without assuming any smallness of initial data.

REMARK 2.4. In the case when $\gamma=2$, a similar result is obtained in a recent interesting paper [8] by Giga and Sawada. For general $\gamma \in(1,2]$, it seems that a direct application of their method only gives a less satisfactory estimate

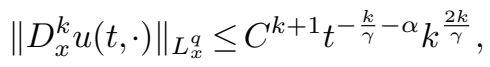

which doesn't imply the spatial analyticity of $u$ if $\gamma<2$. To overcome this difficulty, in the spirit of our previous work [3] we use a fractional bootstrap argument to make better use of the smoothing effect of the corresponding linear equations (see the proof of Theorem 2.1 in section 4). In some sense, our proof can be understood as a nontrivial extension of [8].

The next theorem covers the borderline case $q=d /(\gamma-1)$. It follows from Proposition 2.1 and Theorem 2.1. However, we are not able to obtain a precise analyticity rate estimate as in (2.4).

Theorem 2.5. Let $\gamma \in(1,2]$. Suppose $u$ is a solution to $(2.2)$ in $\mathbb{R}^{d} \times(0, T)$ for some $T \in(0, \infty)$ and satisfies $u \in C\left([0, T), E^{d /(\gamma-1)}\right)$. Then $u(t, \cdot)$ is spatially analytic for any $t \in(0, T)$.

By using a classical argument, it is easy to see that for any pair $(p, q)$ satisfying Serrin's condition

$$
p \in\left(\frac{\gamma}{\gamma-1}, \infty\right), \quad q \in\left(\frac{d}{\gamma-1}, \infty\right), \quad \frac{\gamma}{p}+\frac{d}{q}=\gamma-1,
$$


the solution $u$ in Proposition 2.1 is also in $L_{t}^{q}((0, T)) L_{x}^{p}$ for a possibly different $T>0$. Without much more work, by using a similar proof to that of Theorem 2.1 we can obtain the following result. We omit the details of the proof and leave them to interested readers. The theorem is in the same spirit as a result in Kahane [11], but with equality in Serrin's condition instead of inequality (see also Dong and Du [4]). To the best of our knowledge, the result is new even for the classical Navier-Stokes equations, i.e., $\gamma=2$. Moreover, the smoothing estimate presented here is optimal.

TheOREm 2.6. Let $\gamma \in(1,2]$. Suppose $u$ is a solution to $(2.2)$ in $\mathbb{R}^{d} \times(0, T)$ for some $T \in(0, \infty]$, and satisfies

$$
\|u\|_{L_{t}^{p}((0, T)) L_{x}^{q}<\infty}
$$

for some pair $(p, q)$ satisfying $(2.5)$. Then for any $k=0,1,2, \cdots$ and pair $\left(p^{\prime}, q^{\prime}\right)$ satisfying (2.5) and $q^{\prime} \in[q, \infty)$, we have

$$
\left\|t^{\frac{k}{\gamma}} D_{x}^{k} u\right\|_{L_{t}^{p^{\prime}}((0, T)) L_{x}^{q^{\prime}}} \leq C^{k+1} k^{k},
$$

where $C$ is independent of $k$ and $q^{\prime}$. Consequently, by using the Sobolev embedding theorem, we conclude that $u(t, \cdot)$ is spatially analytic. Moreover, if $f$ is conservative, then $T$ may be infinite.

REMARK 2.7. With some minor modifications our proofs also apply to the periodic boundary condition case. We again leave the details to interested readers.

\section{Pointwise estimates of the generalized Oseen kernel}

We will need the following pointwise estimates of higher derivatives of the generalized Oseen kernel with an explicit control of constants, which we are unable to find in the literature. The proof of a similar result but with no control of constants can be found in [19, Prop. 11.1]. We will not use Proposition 3.1 in its full generality. However, the estimate itself is of independent interest and we present it for future reference. We set $\Lambda=(-\Delta)^{1 / 2}$.

Proposition 3.1. Assume $d \geq 2$ and $\gamma \in(0, \infty)$. For $1 \leq j, m<d$, and $t>0$, the operator $O_{j, m, t}=\frac{1}{\Delta} D_{j} D_{m} e^{-t \Lambda^{\gamma}}$ is a convolution operator whose kernel is given by

$$
K_{j, m, t}=\frac{1}{t^{d / \gamma}} K_{j, m}\left(\frac{x}{t^{1 / \gamma}}\right),
$$

where $K_{j, m}$ is a smooth function. There exists a constant $C=C(d, \alpha, \gamma)$ such that, for any integer $k \geq 0$ and $-1<\alpha \leq 1$ satisfying $d+\alpha>2$,

$$
\left|(1+|x|)^{d+k+\alpha} D^{k} \Lambda^{\alpha} K_{j, m}\right| \leq C^{k+1} k^{k} \quad \forall x \in \mathbb{R}^{d} .
$$

Proof. Let $G_{\gamma}(t, y)$ be the generalized heat kernel which satisfies the scaling property (2.1). Consider first the case $0 \leq|x| \leq 1$. We have

$$
\begin{aligned}
& \max _{0 \leq|x| \leq 1}\left|D_{x}^{k} \Lambda^{\alpha} K_{j, m}\right| \leq \max _{0 \leq|x| \leq 1}\left|D_{x}^{k+2} \Lambda^{\alpha-2} G_{\gamma}(1, x)\right| \\
& \leq\left\|D_{x}^{k+2} \Lambda^{\alpha-2} G_{\gamma}(1, \cdot)\right\|_{L_{x}^{\infty}} \leq \int_{\mathbb{R}^{d}}|\xi|^{k+\alpha} e^{-|\xi|^{\gamma}} d \xi \leq C^{k+1} k^{k} .
\end{aligned}
$$


Now it is enough to show that $\forall|x| \geq 1$, we have

$$
D_{x}^{k+2} \Lambda^{\alpha} \int \frac{1}{|x-y|^{d-2}} G_{\gamma}(1, y) d y \leq \frac{C^{k+1} k^{k}}{|x|^{k+\alpha+d}}
$$

To this end, write $x=t^{-1 / \gamma} \hat{n}, \hat{n} \in S^{d-1}, y=t^{-1 / \gamma} z, 0<t \leq 1$, and we have

$$
\begin{aligned}
& |x|^{k+\alpha+d} D_{x}^{k+2} \Lambda^{\alpha} \int \frac{1}{|x-y|^{d-2}} G_{\gamma}(1, y) d y \\
= & C_{1}|x|^{k+\alpha+d} \int \frac{1}{|x-y|^{d-2+\alpha}} D_{y}^{k+2} G_{\gamma}(1, y) d y \\
= & C_{1} \int \frac{1}{|\hat{n}-z|^{d-2+\alpha}} D_{z}^{k+2} G_{\gamma}(t, z) d z,
\end{aligned}
$$

where $C_{1}=C_{1}(d, \gamma, \alpha)$ is another constant.

Now note that as $t \rightarrow 0, G_{\gamma}(t, x) \rightarrow \delta_{0}(x)$, where $\delta_{0}$ is the Dirac distribution on $\mathbb{R}^{d}$. Therefore it is easy to see that the right-hand side of the above converges in the sense of distributions to

$$
\left.D_{z}^{k+2}\left(\frac{1}{|\hat{n}-z|^{d-2+\alpha}}\right)\right|_{z=0}
$$

Clearly this is bounded by $C^{k+1} \cdot k^{k}$ for some constant $C>0$. We remark that this heuristic argument suggests why the optimal bound on the constants is of the form $C^{k+1} \cdot k^{k}$.

To complete our argument, we write

$$
\begin{aligned}
& \int \frac{1}{|\hat{n}-z|^{d-2+\alpha}} D_{z}^{k+2} G_{\gamma}(t, z) d z \\
= & \int_{|\hat{n}-z| \leq 1 / 2} \frac{1}{|\hat{n}-z|^{d-2+\alpha}} D_{z}^{k+2} G_{\gamma}(t, z) d z \\
& +\int_{|\hat{n}-z|>1 / 2} \frac{1}{|\hat{n}-z|^{d-2+\alpha}} D_{z}^{k+2} G_{\gamma}(t, z) d z \\
= & \mathrm{I}+\mathrm{II} .
\end{aligned}
$$

To estimate I, we use the representation of $G_{\gamma}(t, z)$ through the heat kernel:

$$
G_{\gamma}(t, z)=\int_{0}^{\infty} \frac{1}{\left(\pi^{\frac{1}{2}} s^{\frac{1}{2}} t^{\frac{1}{\gamma}}\right)^{d}} \exp \left\{-\left|\frac{z}{s^{\frac{1}{2}} t^{\frac{1}{\gamma}}}\right|^{2}\right\} d F(s)
$$


where $d F(\cdot)$ is a certain probability measure $[25]^{3}$. This gives us

$$
\begin{aligned}
& D_{z}^{k+2} G_{\gamma}(t, z) \\
= & \int_{0}^{\infty} \frac{1}{\left(\pi^{\frac{1}{2}} s^{\frac{1}{2}} t^{\frac{1}{\gamma}}\right)^{d}} D_{z}^{k+2} \exp \left\{-\left|\frac{z}{s^{\frac{1}{2}} t^{\frac{1}{\gamma}}}\right|^{2}\right\} d F(s) \\
= & \int_{0}^{\infty} \pi^{-\frac{d}{2}}\left(t^{\frac{1}{\gamma}} s^{\frac{1}{2}}\right)^{-(k+d+2)} \cdot(-1)^{k} \cdot H e_{k+2}\left(\frac{z}{s^{\frac{1}{2}} t^{\frac{1}{\gamma}}}\right) \\
& \cdot \exp \left\{-\left|\frac{z}{s^{\frac{1}{2}} t^{\frac{1}{\gamma}}}\right|^{2}\right\} d F(s),
\end{aligned}
$$

where $\mathrm{He}_{k+2}(z)$ is the $d$-dimensional Hermite polynomial of degree $k+2$. We now use the following pointwise estimate of Hermite polynomials [10]:

$$
\left|H e_{n}(x)\right| \leq\left(2^{n} n !\right)^{\frac{1}{2}} e^{\frac{x^{2}}{2}} .
$$

Then we have

$$
\begin{aligned}
& \left|D_{z}^{k+2} G_{\gamma}(t, z)\right| \\
\leq & \int_{0}^{\infty}\left(t^{\frac{1}{\gamma}} s^{\frac{1}{2}}\right)^{-(k+d+2)} \cdot 2^{\frac{k}{2}+1} \cdot((k+2) !)^{\frac{1}{2}} \cdot \exp \left\{-\frac{1}{2}\left|\frac{z}{s^{\frac{1}{2}} t^{\frac{1}{\gamma}}}\right|^{2}\right\} d F(s) .
\end{aligned}
$$

Since in case I, $|\hat{n}-z| \leq \frac{1}{2}$ and therefore $|z| \geq \frac{1}{2}$, we have

$$
\begin{aligned}
& \left|D_{z}^{k+2} G_{\gamma}(t, z)\right| \\
\leq & C^{k+1} \cdot((k+2) !)^{\frac{1}{2}} \int_{0}^{\infty}\left(t^{\frac{1}{\gamma}} s^{\frac{1}{2}}\right)^{-(k+d+2)} \exp \left\{-\frac{1}{8\left|s^{\frac{1}{2}} t^{\frac{1}{\gamma}}\right|^{2}}\right\} d F(s) \\
\leq & C^{k+1} k^{k}
\end{aligned}
$$

where the last inequality follows from the fact that $d F(s)$ is a probability measure and the elementary inequality

$$
\sup _{x>0} x^{k+d+2} e^{-\frac{x^{2}}{8}} \leq C^{k+1} \cdot k^{\frac{k}{2}} .
$$

The estimate of II is similar. By using integration by parts and (3.1) we have,

$$
\begin{aligned}
|\mathrm{II}| & \leq \sum_{j=0}^{k+1} \int_{0}^{\infty}\left(s^{\frac{1}{2}} t^{\frac{1}{\gamma}}\right)^{-d} \int_{|z-\hat{n}|=\frac{1}{2}}\left|D_{z}^{j}\left(\frac{1}{|z-\hat{n}|^{d-2+\alpha}}\right)\right| \\
& \cdot\left|D_{z}^{k-j+1} \exp \left\{-\left|\frac{z}{s^{\frac{1}{2}} t^{\frac{1}{\gamma}}}\right|^{2}\right\}\right| d \sigma(z) d F(s)+ \\
& +\int_{0}^{\infty}\left(s^{\frac{1}{2}} t^{\frac{1}{\gamma}}\right)^{-d} \int_{|z-\hat{n}|>\frac{1}{2}}\left|D_{z}^{k+2}\left(\frac{1}{|z-\hat{n}|^{d-2+\alpha}}\right)\right| \exp \left\{-\left|\frac{z}{s^{\frac{1}{2}} t^{\frac{1}{\gamma}}}\right|^{2}\right\} d z d F(s)
\end{aligned}
$$

\footnotetext{
${ }^{3}$ Our proof does not use the explicit form of $d F$, but only the fact that it is a probability measure.
} 
where $d \sigma$ is the standard measure on the sphere $\{|z-\hat{n}|=1 / 2\}$.

On $|z-\hat{n}|=\frac{1}{2}$, we have $|z| \geq \frac{1}{2}$ and therefore by (3.2)

$$
\begin{aligned}
& \left|D_{z}^{k-j+1} \exp \left\{-\left|\frac{z}{s^{\frac{1}{2}} t^{\frac{1}{\gamma}}}\right|^{2}\right\}\right| \\
= & \left(s^{\frac{1}{2}} t^{\frac{1}{\gamma}}\right)^{-(k-j+1)} \cdot\left|H e_{k-j+1}\left(\frac{z}{s^{\frac{1}{2}} t^{\frac{1}{\gamma}}}\right)\right| \cdot \exp \left\{-\left|\frac{z}{s^{\frac{1}{2}} t^{\frac{1}{\gamma}}}\right|^{2}\right\} \\
\leq & \left(s^{\frac{1}{2}} t^{\frac{1}{\gamma}}\right)^{-(k-j+1)} \cdot 2^{\frac{k-j+1}{2}}((k-j+1) !)^{\frac{1}{2}} \cdot \exp \left\{-\frac{1}{8\left|s^{\frac{1}{2}} t^{\frac{1}{\gamma}}\right|^{2}}\right\} .
\end{aligned}
$$

Also note that for $|z-\hat{n}|>\frac{1}{2}$, we have

$$
\left|D_{z}^{k+2}\left(\frac{1}{|z-\hat{n}|^{d-2+\alpha}}\right)\right| \leq C^{k+1} k ! .
$$

These estimates together with the elementary inequality (3.3) immediately give us

$$
|\mathrm{II}| \leq \sum_{j=0}^{k+1} C^{k+1} j !(k-j+1) !+C^{k+1} k^{k} \leq C^{k+1} k^{k},
$$

where the second inequality follows from the Stirling's formula. The proposition is now proved.

We also need the following lemma.

Lemma 3.1. Let $\gamma \in(0, \infty)$ and $p \in[1, \infty]$. Let $k \geq 0$ be an integer and $\varepsilon \in(0,1]$. Then for some constant $C=C(d, \gamma, \varepsilon)>0$, we have

$$
\left\|D_{x}^{k} \Lambda^{\alpha} G_{\gamma}(t, \cdot)\right\|_{L_{x}^{p}} \leq C^{k+1} k^{\frac{k}{\gamma}} t^{-\frac{k+\alpha}{\gamma}-\frac{d}{\gamma}\left(1-\frac{1}{p}\right)}
$$

for any $\alpha$ satisfying

$$
\begin{cases}\varepsilon-1 \leq \alpha \leq 1 & \text { if } k \geq 1 \\ \varepsilon \leq \alpha \leq 1 \text { or } \alpha=0 & \text { if } k=0 .\end{cases}
$$

Here the constant $C$ can be taken to be independent of $p$.

Proof. This follows from a similar pointwise estimate as in Proposition 3.1. We omit the details.

Corollary 3.2 .

i) Assume $d \geq 2, \gamma \in(0, \infty)$, and $\varepsilon \in(0,1]$. There exists a constant $C=C(d, \varepsilon, \gamma)$ such that for any integer $k \geq 0, p \in[1, \infty]$, and $-1<\alpha \leq 1$ satisfying $d+\alpha \geq 2+\varepsilon$,

$$
\left\|D_{x}^{k} \Lambda^{\alpha} K_{\gamma}(t, \cdot)\right\|_{L_{x}^{p}} \leq C^{k+1} k^{\frac{k}{\gamma}} t^{-\frac{k+\alpha}{\gamma}-\frac{d}{\gamma}\left(1-\frac{1}{p}\right)} .
$$

ii) Assume $d=2, \gamma \in(0, \infty), p \in(1, \infty)$ and $\varepsilon \in(0,1]$. There exists a constant $C=$ $C(\varepsilon, p, \gamma)$ such that (3.5) holds for any integer $k \geq 1$ and $\alpha \in[\varepsilon-1,1]$.

Proof. By scaling, it suffices to consider the case $t=1$. In this case, the corollary follows immediately from Proposition 3.1. 


\section{Proof of Theorem 2.1}

For simplicity of the proofs, we assume that $f$ is conservative. The arguments can be extended to $f$ satisfying (2.3) without any difficulty. Since $u$ is divergence free, we have

$$
u \nabla u=\nabla \cdot(u \otimes u) .
$$

Therefore, by using integration by parts, the integral equation (2.2) is equivalent to

$$
u(t)=G_{\gamma}(t) * u_{0}-B(u, u),
$$

where

$$
B(u, u):=\int_{0}^{t} \nabla K_{\gamma}(t-s, \cdot) *(u \otimes u)(s, \cdot) d s
$$

is a bilinear term. The following lemma is probably known. We provide a sketched proof for the sake of completeness.

Lemma 4.1. Under the assumptions of Theorem 2.1, for any $t \in(0, T)$ and $q^{\prime} \in[q, \infty]$ we have

$$
\|u(t, \cdot)\|_{L_{x}^{q^{\prime}}} \leq C t^{-\alpha^{\prime}}
$$

where $\alpha^{\prime}=1-\frac{1}{\gamma}-\frac{d}{q^{\prime} \gamma}$ and the constant $C$ is independent of $q^{\prime}$.

Proof. In this proof we denote the constants which may vary from line to line but which are independent of $q^{\prime}$ by $C$. We shall use a bootstrap argument. Assume for some positive constant $C_{0}$ that

$$
\left\|t^{\alpha}\right\| u(t, \cdot)\left\|_{L_{x}^{q}}\right\|_{L_{t}^{\infty}(0, T)} \leq C_{0} .
$$

We fix a $t \in(0, T)$ and choose $s \in(t / 3,2 t / 3)$ such that

$$
s^{\alpha}\|u(s, \cdot)\|_{L_{x}^{q}} \leq C_{0} .
$$

From (4.1) and the semigroup property of $G_{\gamma}$, it holds that

$$
u(t, \cdot)=G_{\gamma}(t-s) * u(s, \cdot)-\int_{s}^{t} \nabla K_{\gamma}(t-r, \cdot) *(u \otimes u)(r, \cdot) d r .
$$

Taking the $L_{x}^{q^{\prime}}$ norm on both sides and using Minkowski's inequality, Young's inequality, Hölder's inequality, Proposition 3.1, and Lemma 3.1, we obtain

$$
\begin{aligned}
& \|u(t, \cdot)\|_{L^{q^{\prime}}} \\
\leq & \left\|G_{\gamma}(t-s) * u(s, \cdot)\right\|_{L^{q^{\prime}}}+\int_{s}^{t}\left\|\nabla K_{\gamma}(t-r, \cdot) *(u \otimes u)(r, \cdot)\right\|_{L^{q^{\prime}}} d r \\
\leq & \left\|G_{\gamma}(t-s)\right\|_{L^{r_{1}}}\|u(s, \cdot)\|_{L^{q}}+\int_{s}^{t}\left\|\nabla K_{\gamma}(t-r, \cdot)\right\|_{L^{r_{2}}}\|u(r, \cdot)\|_{L^{q}}^{2} d r \\
\leq & C(t-s)^{-\frac{d}{\gamma}\left(1-\frac{1}{r_{1}}\right)} s^{-\alpha}+C \int_{s}^{t}(t-r)^{-\frac{1}{\gamma}-\frac{d}{\gamma}\left(1-\frac{1}{r_{2}}\right)} r^{-2 \alpha} d r,
\end{aligned}
$$


where $r_{1}$ and $r_{2}$ satisfy

$$
1+\frac{1}{q^{\prime}}=\frac{1}{r_{1}}+\frac{1}{q}, \quad 1+\frac{1}{q^{\prime}}=\frac{1}{r_{2}}+\frac{2}{q}
$$

Because $s \in(t / 3,2 t / 3)$,

$$
\|u(t, \cdot)\|_{L^{q^{\prime}}} \leq C t^{-\alpha^{\prime}}+C t^{-\alpha^{\prime}} \int_{1 / 3}^{1}(1-r)^{-\frac{1}{\gamma}-\frac{d}{\gamma}\left(1-\frac{1}{r_{2}}\right)} r^{-2 \alpha} d r .
$$

Since $q>\frac{d}{\gamma-1}$, the last integral is finite if

$$
0 \leq \frac{1}{q}-\frac{1}{q^{\prime}} \leq \frac{1}{2 d}\left(\gamma-1-\frac{d}{q}\right)
$$

Hence, (4.2) is proved for $q^{\prime}$ satisfying (4.3). A finite iteration of this argument gives (4.3) for any $q^{\prime} \in[q, \infty]$. The lemma is proved.

Now we are ready to prove Theorem 2.1.

Proof of Theorem 2.1. In this proof we shall denote constants which may vary from line to line but which do not depend on $k$ or $q^{\prime}$ by $C_{1}$. Let $N \geq 1$ be an integer sufficiently large such that

$$
1+\frac{1}{N}+\frac{2 d}{N q}+\frac{d}{q}<\gamma
$$

Define a finite sequence of numbers $q_{n} \geq q$ such that

$$
\frac{1}{q_{n}}=\frac{1}{q}-\frac{n}{N q}, \quad 0 \leq n \leq N .
$$

Also define, for $l=0,1, \ldots, N-1$, and $q^{\prime} \in[q, \infty]$,

$$
A\left(k, l, q^{\prime}\right)=\left\|t^{\alpha^{\prime}+\frac{k+l / N}{\gamma}} D_{x}^{k} \Lambda^{\frac{l}{N}} u\right\|_{L_{t}^{\infty}(0, T) L_{x}^{q^{\prime}}}
$$

and

$$
A(k, l)=\sup _{q \leq q^{\prime} \leq \infty} A\left(k, l, q^{\prime}\right)
$$

We shall derive a set of recurrent inequalities for $A(k, l)$. To this end, by using the semigroup property of $G_{\gamma}$, write

$$
u(t, \cdot)=G_{\gamma}\left(\frac{t}{k+2}\right) * u\left(\frac{k+1}{k+2} t\right)-\int_{\frac{k+1}{k+2} t}^{t} \nabla K_{\gamma}(t-r, \cdot) *(u \otimes u)(r, \cdot) d r .
$$

Call the first term in the above sum the linear term and the other the bilinear term. We have four cases. 
Case 1: estimate of the linear term for $k \geq 0,1 \leq l \leq N-1$. By Lemma 3.1 we have

$$
\begin{aligned}
& \left\|t^{\alpha^{\prime}+\frac{k+l / N}{\gamma}} D_{x}^{k} \Lambda^{\frac{l}{N}}\left(G_{\gamma}\left(\frac{t}{k+2}\right) * u\left(\frac{k+1}{k+2} t\right)\right)\right\|_{L_{t}^{\infty} L_{x}^{q^{\prime}}} \\
\leq & \left\|t^{\frac{1}{N \gamma}} \Lambda^{\frac{1}{N}} G_{\gamma}\left(\frac{t}{k+2}\right)\right\|_{L_{t}^{\infty} L_{x}^{1}}\left\|t^{\alpha^{\prime}+\frac{k+\frac{l-1}{N}}{\gamma}} D_{x}^{k} \Lambda^{\frac{l-1}{N}} u\left(\frac{k+1}{k+2} t\right)\right\|_{L_{t}^{\infty} L_{x}^{q^{\prime}}} \\
\leq & C_{1}(k+2)^{\frac{1}{N \gamma}} \cdot\left(\frac{k+2}{k+1}\right)^{\alpha^{\prime}+\frac{k+\frac{l-1}{N}}{\gamma}} A(k, l-1) \\
\leq & C_{1}(k+1)^{\frac{1}{N \gamma}} A(k, l-1) .
\end{aligned}
$$

Case 2: estimate of the linear term for $l=0, k \geq 1$. This case is similar to Case 1 and we have

$$
\begin{aligned}
& \left\|t^{\alpha^{\prime}+\frac{k}{\gamma}} D_{x}^{k}\left(G_{\gamma}\left(\frac{t}{k+2}\right) * u\left(\frac{k+1}{k+2} t\right)\right)\right\|_{L_{t}^{\infty} L_{x}^{q^{\prime}}} \\
= & \left\|t^{\alpha^{\prime}+\frac{k}{\gamma}} \Lambda^{\frac{1}{N}} \Lambda^{-1} D_{x} G_{\gamma}\left(\frac{t}{k+2}\right) * D_{x}^{k-1} \Lambda^{\frac{N-1}{N}} u\left(\frac{k+1}{k+2} t\right)\right\|_{L_{t}^{\infty} L_{x}^{q^{\prime}}} \\
\leq & C_{1} \cdot(k+1)^{\frac{1}{N \gamma}} A(k-1, N-1) .
\end{aligned}
$$

Case 3: estimate of the nonlinear term for $k \geq 0,1 \leq l \leq N-1$. Consider $q^{\prime} \in$ $[q, \infty]$, where obviously $\frac{1}{q^{\prime}} \in\left[\frac{1}{q_{n+1}}, \frac{1}{q_{n}}\right]$ for some $0 \leq n \leq N-1$. For any two functions $f, g$, and $0 \leq \varepsilon<1,2<p<\infty$, the following fractional Leibniz inequality is well known:

$$
\left\|\Lambda^{\varepsilon}(f g)\right\|_{L_{x}^{p / 2}} \leq C_{p}\left(\left\|\Lambda^{\varepsilon} f\right\|_{L_{x}^{p}}\|g\|_{L_{x}^{p}}+\left\|\Lambda^{\varepsilon} g\right\|_{L_{x}^{p}}\|f\|_{L_{x}^{p}}\right),
$$

where the constant $C_{p}$ depends on $p$ (see, for instance, [14]). In what follows, we shall only apply the fractional Leibniz inequality when $p=q_{n}$ for $0 \leq n \leq N-1$. In this way the constants will not depend on $q^{\prime}$. Now by Corollary 3.2, Young's inequality, and the fractional Leibniz inequality, we have

$$
\begin{aligned}
& \left\|t^{\alpha^{\prime}+\frac{k+\frac{l}{N}}{\gamma}} D_{x}^{k} \Lambda^{\frac{l}{N}} \int_{\frac{k+1}{k+2} t}^{t} \nabla K_{\gamma}(t-r, \cdot) *(u \otimes u)(r) d r\right\|_{L_{t}^{\infty} L_{x}^{q^{\prime}}} \\
= & \left\|t^{\alpha^{\prime}+\frac{k+\frac{l}{N}}{\gamma}} \int_{\frac{k+1}{k+2} t}^{t} \Lambda^{\frac{1}{N}} \nabla K_{\gamma}(t-r, \cdot) *\left(D_{x}^{k} \Lambda^{\frac{l-1}{N}}(u \otimes u)(r)\right) d r\right\|_{L_{t}^{\infty} L_{x}^{q^{\prime}}} \\
\leq & C_{1}\left(\int_{1-\frac{1}{k+2}}^{1}(1-r)^{-\frac{1+\frac{1}{N}}{\gamma}-\frac{d}{\gamma}\left(\frac{2}{q_{n}}-\frac{1}{q^{\prime}}\right)} d r\right) \sum_{j=0}^{k}\left(\begin{array}{c}
k \\
j
\end{array}\right) . \\
& \cdot\left(1-\frac{1}{k+2}\right)^{-\frac{k+\frac{l-1}{\gamma}}{\gamma}-\left(2-\frac{2}{\gamma}-\frac{2 d}{\gamma q_{n}}\right)} \cdot A\left(j, l-1, q_{n}\right) A\left(k-j, 0, q_{n}\right) \\
\leq & C_{1} \sum_{j=0}^{k}\left(\begin{array}{c}
k \\
j
\end{array}\right) A(j, l-1) A(k-j, 0) .
\end{aligned}
$$

The last integral converges since we have

$$
\frac{1+\frac{1}{N}}{\gamma}+\frac{d}{\gamma}\left(\frac{2}{q_{n}}-\frac{1}{q^{\prime}}\right) \leq \frac{1+\frac{1}{N}}{\gamma}+\frac{d}{\gamma q}+\frac{d}{N q \gamma}<1
$$


Case 4: estimate of the nonlinear term for $k \geq 1$ and $l=0$. This case is similar to Case 3 but slightly trickier. The trick is to write

$$
\begin{aligned}
& D_{x}^{k} \int_{\frac{k+1}{k+2} t}^{t} \nabla K_{\gamma}(t-r, \cdot) *(u \otimes u)(r) d r \\
= & \int_{\frac{k+1}{k+2} t}^{t} D_{x} \Lambda^{-\frac{N-1}{N}} \nabla K_{\gamma}(t-r, \cdot) * D_{x}^{k-1} \Lambda^{\frac{N-1}{N}}(u \otimes u)(r) d r .
\end{aligned}
$$

Now the rest of the proof follows essentially the same line as in Case 3. We have

$$
\begin{aligned}
& \left\|t^{\alpha^{\prime}+\frac{k}{\gamma}} D_{x}^{k} \int_{\frac{k+1}{k+2} t}^{t} \nabla K_{\gamma}(t-r, \cdot) *(u \otimes u)(r) d r\right\|_{L_{t}^{\infty} L_{x}^{q^{\prime}}} \\
\leq & C_{1} \sum_{j=0}^{k-1}\left(\begin{array}{c}
k-1 \\
j
\end{array}\right) A(j, N-1) A(k-1-j, 0) .
\end{aligned}
$$

Concluding from the above four cases, and by Lemma 4.1, we have the following recurrent inequalities for $A(k, l)$.

For $k=0, l=0$,

$$
A(0,0) \leq C .
$$

For $k \geq 0,1 \leq l \leq N$, denote $A(k, N)=A(k+1,0)$, and we have

$$
A(k, l) \leq C_{1}(k+1)^{\frac{1}{N \gamma}} A(k, l-1)+C_{1} \sum_{j=0}^{k}\left(\begin{array}{l}
k \\
j
\end{array}\right) A(j, l-1) A(k-j, 0) .
$$

Here $C$ and $C_{1}$ are constants greater than 1 . For $k \geq 0,1 \leq l \leq N, 0 \leq j \leq k$, denote

$$
n_{1}=N j+l-1, \quad n_{2}=N(k-j), \quad n=N k+l .
$$

The following inequality is easy to prove by using Stirling's formula:

$$
\left(\begin{array}{c}
k \\
j
\end{array}\right) \leq C_{1}\left(\frac{n^{n}}{n_{1}^{n_{1}} n_{2}^{n_{2}}}\right)^{\frac{1}{N}} .
$$

Then it is not difficult to see that

$$
A(k, l) \leq F(N k+l),
$$

where $F(n)$ is a sequence of numbers satisfying

$$
F(0) \leq C,
$$

and for $n \geq 1$,

$$
\begin{aligned}
F(n)= & C_{1} n^{\frac{1}{N \gamma}} F(n-1) \\
& +C_{1} \sum_{n_{1}=0}^{n-1} \frac{n^{n / N}}{n_{1}^{n_{1} / N}\left(n-1-n_{1}\right)^{\left(n-1-n_{1}\right) / N}} F\left(n_{1}\right) F\left(n-1-n_{1}\right) .
\end{aligned}
$$


Clearly $F(n) \leq\left(C_{1} C\right)^{n+1} n^{n / N} G(n)$, where $G(0)=1$ and

$$
G(n)=2 \sum_{n_{1}=0}^{n-1} G\left(n_{1}\right) G\left(n-1-n_{1}\right)
$$

By the method of formal power series, it is easy to show that for some constant $C>0$,

$$
G(n) \leq C^{n+1} \text {. }
$$

This immediately yields that

$$
A(k, l) \leq C^{k+1} k^{k}
$$

Our theorem is proved.

\section{Proof of Theorem 2.5}

This section is devoted to the proof of Theorem 2.5. We need the following uniqueness result of the mild solution to (1.1).

Lemma 5.1. The solution to $(2.2)$ in $C\left(\left[0, T_{1}\right), E^{d /(\gamma-1)}\right)$ is unique for any $T_{1} \in(0, \infty]$.

Indeed, for $\gamma=2$ this lemma is proved in [23] (see also [17]). The proof there can be easily modified to cover the case $\gamma \in(1,2]$. We leave the details to interested readers.

Now we are ready to prove Theorem 2.5. We fix a $t \in(0, T)$ and $q \in\left(\frac{d}{\gamma-1}, \infty\right)$. For any $s \in[0, t]$, by Proposition 2.1 there exists a $\varepsilon_{s} \in(0, T-s)$ and a solution $v_{s} \in X_{q, \varepsilon_{s}}$ to (2.2). Furthermore, $\varepsilon_{s}$ depends continuously on $u(s, \cdot)$ in $E^{\frac{d}{\gamma-1}}$-topology. Under the assumption of Theorem 2.5, we can find a uniform lower bound $\varepsilon=\inf [0, t] \varepsilon_{s}$. Theorem 2.1 implies that $v_{s}(r, \cdot)$ is spatially analytic for any $r \in(0, \varepsilon)$. Due to Lemma 5.1 , we know that

$$
v_{s}(r, \cdot)=u(s+r, \cdot) \quad \forall s \in[0, t], r \in[0, \varepsilon) .
$$

In particular, this shows that $u(t, \cdot)$ is spatially analytic. The theorem is proved.

Acknowledgement. H. Dong was partially supported by a start-up funding from the Division of Applied Mathematics of Brown University and the National Science Foundation under agreement No. DMS-0111298 and DMS-0800129. D. Li was partially supported by a start-up funding from the Mathematics Department of University of Iowa and the National Science Foundation under agreement No. DMS0111298 and DMS-0635607. The authors would like to thank the referees for their very helpful comments on the first submission of the article.

\section{REFERENCES}

[1] C. Taubes, The Seiberg-Witten invariants and symplectic forms, Math. Res. Letters, 1, 809822, 1994.

[2] J.A. Carrilo and L.C.F. Ferreira, The asymptotic behavior for the subcritical dissipative quasigeostrophic equations, Nonlinearity, 21, 1001-1018, 2008.

[3] H. Dong and D. Li, Spatial analyticity of the solutions to the sub-critical dissipative quasigeostrophic equations, Arch. Rational Mech. Anal., 189(1), 131-158, 2008.

[4] H. Dong and D. Du, On the local smoothness of solutions of the Navier-Stokes equations, J. Math. Fluid Mech., 9(2), 139-152, 2007. 
[5] C. Foias and R. Temam, Gevrey class regularity for the solutions of the Navier-Stokes equations, J. Funct. Anal., 87, 359-369, 1989.

[6] I. Gallagher, D. Iftimie and F. Planchon, Asymptotics and stability for global solutions to the Navier-Stokes equations, Ann. Inst. Fourier (Grenoble), 53(5), 1387-1424, 2003.

[7] P. Germain, N. Pavlović and G. Staffilani, Regularity of solutions to the Navier-Stokes equations evolving from small data in $B M O^{-1}$, Int. Math. Res. Not., Art. ID rnm087, 35(21), 2007.

[8] Y. Giga and O. Sawada, On regularizing-decay rate estimates for solutions to the Navier-Stokes initial value problem, Nonlinear analysis and applications: to V. Lakshmikantham on his 80th birthday, Kluwer Acad. Publ., Dordrecht, 1(2), 549-562, 2003.

[9] Z. Grujić and I. Kukavica, Space analyticity for the Navier-Stokes and related equations with initial data in $L^{p}$, J. Funct. Anal., 152, 447-466, 1998.

[10] J. Indritz, An inequality for Hermite polynomials, Proc. Amer. Math. Soc., 12(6), 981-983, 1961.

[11] C. Kahane, On the spatial analyticity of solutions of the Navier-Stokes equations, Arch. Ration. Mech. Anal., 33, 386-405, 1969.

[12] N.H. Katz and N. Pavlović, A cheap Caffarelli-Kohn-Nirenberg inequality for the Navier-Stokes equation with hyper-dissipation, Geom. Funct. Anal., 12(2), 355-379, 2002.

[13] T. Kato, Strong $L^{p}$-solutions of the Navier-Stokes equation in $\mathbb{R}^{m}$ with applications to weak solutions, Math. Z., 187, 471-480, 1984.

[14] C. Kenig, G. Ponce and L. Vega, Well-posedness of the initial value problem for the KortewegDe Vries equation, J. Amer. Math. Soc., 4, 323-347, 1991.

[15] H. Koch and D. Tataru, Well-posedness for the Navier-Stokes equations, Adv. Math., 157(1), $22-35,2001$.

[16] G. Komatsu, Global analyticity up to the boundary of solutions of the Navier-Stokes equation, Comm. Pure Appl. Math., 33, no. 4, 545-566, 1980.

[17] G. Furioli, P.G. Lemarié-Rieusset and E. Terraneo, Sur l'unicité dans $L^{3} 3\left(\mathbb{R}^{3}\right)$ des solutions "mild" des équations de Navier-Stokes. (French) [On the uniqueness in $L^{3}\left(\mathbb{R}^{3}\right)$ of mild solutions for the Navier-Stokes equations], C.R. Acad. Sci. Paris Série I Math., 325(12), 1253-1256, 1997.

[18] P.G. Lemarié-Rieusset, Une remarque sur lánalyticity des solutions milds des équations de Navi er-Stokes dans $\mathbb{R}^{3}$, R. Acad. Sci. Paris, 330, Série I, 183-186, 2000.

[19] P.G. Lemarié-Rieusset, Recent Developments in the Navier-Stokes Problem, Chapman \& Hall/CRC, 2002.

[20] P.G. Lemarié-Rieusset, Nouvelles remarques sur l'analyticité des solutions milds des équations de Navier-Stokes dans $\mathbb{R}^{3}$, (French. English, French summary), [Further remarks on the analyticity of mild solutions for the Navier-Stokes equations in $\mathbb{R}^{3} 3$ ], C.R. Math. Acad. Sci. Paris, 338(6), 443-446, 2004.

[21] J.L. Lions, Quelques Méthodes de Réolution des Problèmes aux Limites Non Linéaires, (French) Paris: Dunod / Gauthier-Villars, 1969.

[22] K. Masuda, On the analyticity and the unique continuation theorem for solutions of the NavierStokes equation, Proc. Japan Acad., 43, 827-832, 1967.

[23] S. Monniaux, Uniqueness of mild solutions of the Navier-Stokes equation and maximal $L_{p^{-}}$ regularity, C.R. Acad. Sci. Paris., 328, Série I Math., 663-668, 1999.

[24] H. Miura and O. Sawada, On the regularizing rate estimates of Koch-Tatarus solution to the Navier-Stokes equations, Asymptotic Analysis, 49(1-2), 1-15, 2006.

[25] S. Renick, Dynamical problems in non-linear advective partial differential equations, $\mathrm{PhD}$ thesis, University of Chicago, 1995.

[26] O. Sawada, On analyticity rate estimates of the solutions to the Navier-Stokes equations in Bessel-potential spaces, J. Math. Anal. Appl., 312(1), 1-13, 2005.

[27] J. Wu, Lower bounds for an integral involving fractional Laplacians and the generalized NavierStokes equations in Besov spaces, Commun. Math. Phys., 263(3), 803-831, 2006.

[28] J. Wu, The generalized incompressible Navier-Stokes equations in Besov spaces, Dyn. Partial Differ. Equ., 1(4), 381-400, 2004. 